Le Comité de la section belge a fixé le montant des cotisations pour les membres ordinaires à 150 fr.; la section admet en outre des membres protecteurs dont la cotisation est laissée à l'appréciation et à la générosité de ces membres. Toutes les cotisations peuvent être versées au C.C.P. no 720588 de la section belge de l'Association Internationale de Droit Africain.

(Communication du Dr. J. Vanderlinden)

\title{
L'Institut de Sociologie Solvay: Création du Centre d'Histoire et d'Ethnologie Juridiques
}

L'Instrutut de Sociologie Solvay vient de créer un Centre d'Histoire et d'Ethnologie juridiques placé sous la direction du Professeur J. Gilissen. Le but de la nouvelle institution est de promouvoir les recherches sur l'évolution générale du droit et des institutions, depuis l'antiquité jusqu'à nos jours, ainsi que sur les droits coutumiers des peuples primitifs.

Les activités du Centre consistent d'une part dans l'élaboration d'instruments de travail tels que bibliographie, répertoires des sources juridiques, etc., d'autre part dans l'organisation de colloques et de séminaires sur les divers aspects de l'évolution du droit. Il est dès à présent envisagé d'organiser un colloque international sur les méthodes de rédaction des coutumes, en vue de comparer les méthodes adoptées jadis en Europe et en Amérique et actuellement en Afrique et en Asie. Enfin, par des missions de recherche en Afrique Centrale, l'étude des transformations subies par les droits coutumiers au contact du droit européen pourra être systématiquement poursuivie.

\section{Economic Study in Ruanda}

A PROJECT to study a small community in Eastern Ruanda, with emphasis on its economy and its relation to world market economy, is being undertaken by M. Pierre Bettez Gravel under the auspices of the Department of Anthropology of the University of Michigan. Particular attention will be paid to the subsistence role of livestock in the community and to the mechanisms regulating this role.

\section{(Communicated by M. Pierre Bettez Gravel)}

\section{Séminaire en Sciences Humaines de l'IRS AC}

Du 3 à 6 février inclus s'est tenu à Lwiro (Bukavu) le séminaire annuel en sciences humaines de l'IRSAC. Les chercheurs associés de l'Institut y ont présenté les communications suivantes: M. E. Van de Walle, Problèmes de méthode d'étude démographique du RuandaUrundi; M. F. Crine, La Parenté dans l'organisation politique lunda: le Prof. A. Merriam, Preliminary Study of the Political Organization of a Bala Village; Dr. J. Vansina, Dieu sur la terre: Le Roi et la distribution du pouvoir chez les Kuba; M. A. Doutreloux, Le Rôle social et politique du nkisi au Mayumbe; M.l'Abbé A. Kagame, La Cour du Rwanda ancien; le Prof. J. Jacobs, La Nouvelle Orthographe tetela-kusu; le Prof. H. Codère, Recherches en cours au Ruanda; M. M. d'Hertefelt, Acculturation politique au Ruanda; le Prof. A. Coupez, Organisation formelle du dictionnaire rwanda en préparation; le R.P. G. Hulstaert, Règle et exception en Mongo; le R.P. F. Van Moorsel, L'Archéologie de la plaine de Léopoldville; le Prof. L. Baeck, État des recherches économiques au Congo Belge et au Ruanda-Urundi; le Prof. D. Biebuyck, État des recherches en anthropologie culturelle au Congo Belge et au Ruanda-Urundi. Quoique le séminaire n'ait pas eu de thème central il a été centré en pratique autour des problèmes du Ruanda-Urundi et des problèmes de structures politiques. 